\title{
Surface plasmon effect in nanocrystalline copper/DLC composite films by electrodeposition technique
}

\author{
$S$ HUSSAIN and A K PAL* \\ Department of Instrumentation Science, Jadavpur University, Kolkata 700 032, India
}

\begin{abstract}
Composite films of nanocrystalline copper embedded in DLC matrix prepared by electrodeposition technique were studied for their optical properties. Particle size and metal volume fractions were tailored by varying the amount of copper containing salt in the electrolyte. Blue-shift of the surface plasmon resonance peak in the absorbance spectra of the films was observed with the reduction in size and volume fraction of metal particles. Mie theory was found to describe the experimental spectra quite well.
\end{abstract}

Keywords. Nanocomposites; surface plasmon; scattering; Mie theory; M-G theory.

\section{Introduction}

Studies on low dimensional structures have become the subject of various research activities in recent times due to their extraordinary properties. Effect of metal incorporation in diamond like carbon (DLC) films were mainly limited to low friction related tribological applications (Angus et al 1991). Difficulty was felt in the homogeneous dispersion of the metal particles by traditional methods though a new class of technologically important materials originated (Paulean and Thiery 2002). With its wide band gap, DLC films could act as host matrix for metals inclusion in nanocrystalline form revealing interesting optical properties like surface plasmon resonance. In these systems, the metal particles were dispersed in the dielectric medium with different inter-grain distances by changing the number density of the ultrafine metal particles. Here, we present our studies on the optical properties of a composite material composed of ultra-fine copper particles embedded in DLC matrix prepared by simple electrodeposition technique.

\section{Experimental}

The composite films of nanocrystalline copper particles in DLC matrix were prepared by electrodeposition as has been reported earlier for DLC films (Gupta et al 2003). Fixed volume percent of acetic acid $\left(\mathrm{CH}_{3} \mathrm{COOH}\right)$ $(1 \% \mathrm{v} / \mathrm{v})$ in water was used along with different concentrations of copper acetate, $\left(\mathrm{CH}_{3} \mathrm{COOH}\right) \mathrm{Cu}$, as electrolyte with bath temperature at $\sim 300 \mathrm{~K}$. A graphite sheet was used as the anode and was separated from the $\mathrm{SnO}_{2}$ coated glass (substrate) acting as cathode by $\sim 0.7 \mathrm{~cm}$. A d.c. voltage

\footnotetext{
*Author for correspondence (msakp2002@yahoo.co.in)
}

power supply capable of generating stabilized voltage $(0-$ $30 \mathrm{~V} ; 1 \AA$ ) was used. The deposition time was $60 \mathrm{~min}$.

\section{Results and discussion}

The nanocomposite copper/DLC films were extremely adherent to the substrates showing lesser stress. Colour of the films varied from greenish blue to reddish yellow with change in size and volume fraction of copper nanoparticles. Transmittance of the films decreased from $85-65 \%$ with an increase in the volume fraction of copper nanoparticles. Films composed of ultra-fine metal particles embedded in dielectric matrix are generally characterized by the presence of a very large surface to volume ratio of crystallites constituting the film. The grains were separated from each other by distances, which were determined by the volume fraction of the metal particle loading. Properties different from those in the bulk with long range order and interaction effects played a major role in the electronic properties. The interaction of the small metal particles with the external electromagnetic field induced by light results into a coherent oscillation of the conduction (free) electrons (mainly within the surfaces), called the surface plasmon resonance. This surface plasmon resonance largely depends on the particle size, shape, the metallic material and its surroundings i.e. DLC. Volume fraction and size of the copper nanocrystallites were controlled by changing the amount of copper salt and current density for a fixed amount of copper salt, respectively. Increasing the proximity of nanocrystallites favoured coalescence of the nanocrystalites to larger clusters and could be achieved by adjusting the voltage applied and the time of deposition. Increase in copper acetate concentration at a fixed applied voltage also had a similar effect. By varying the amount of copper salt and current density during deposition, films with nearly the same particle size but different metal volume fraction $(f)$, respectively, could be obtained. 

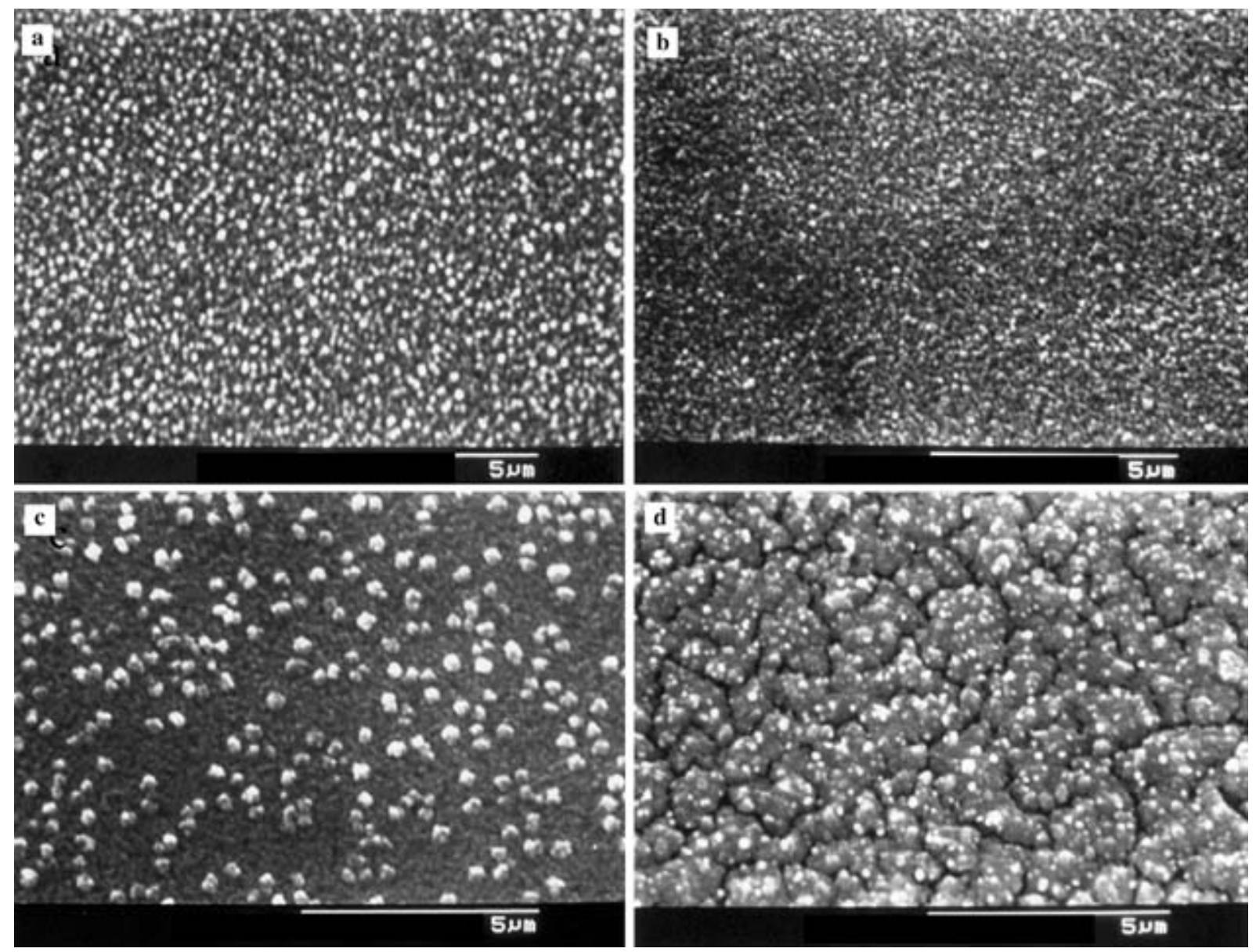

Figure 1. SEM micrographs of a. N/100, $2 \cdot 4 \mathrm{~V}$, b. N/200, $2.4 \mathrm{~V}$, c. N/200, $1.5 \mathrm{~V}$ and d. N/200, $3 \mathrm{~V}$.

\subsection{SEM studies}

SEM micrographs (figures 1a, b) of films deposited at two different concentrations of copper acetate but fixed applied voltage show the films to be compact and granular. The films have the same general topography. Variation of microstructure with change in applied voltage $(1.5,2.4$ and $3.5 \mathrm{~V}$ ), keeping the bath concentration fixed is depicted in figures $1 \mathrm{c}, \mathrm{b}, \mathrm{d}$, respectively. Change in morphologies with variation in sizes and volume fractions are apparent.

\subsection{TEM studies}

TEM micrographs (figure 2) of two films deposited in fixed bath concentration (N/200) but different applied voltages: a. $1.5 \mathrm{~V}$ and b. $3.5 \mathrm{~V}$, show the change in morphology with applied voltage. Particle size increased with higher current density. Increase in the amount of copper acetate in the bath increased the number density and size of the nanocrystallites, thus, broadening the size-distribution of the nanocrystallites. The inter-particle distances became smaller with the increase in number density of particles and particles were still well isolated from each other.
XRD pattern of a representative film (figure 3 ) exhibits features differing significantly from that of coarse grained polycrystalline materials (Grigson and Barton 1967). Peaks are broad in nature with a slight shift in peak value as compared to that of bulk material. The broad peak at $2 \theta=43.3^{\circ}(\theta$ is the Bragg angle $)$ due to the (111) plane of copper with a lattice spacing of $2.09 \AA$ agrees with the corresponding lattice value of the bulk (2.088 $\AA$ ). The crystal sizes obtained from TEM and XRD data agreed reasonably well with each other.

\subsection{Optical studies}

Optical absorbance spectra (figure 4) of the nanocomposite films deposited (a) with different bath concentrations but fixed applied voltage $(2.4 \mathrm{~V})$ and (b) at different applied voltages but fixed bath concentration (N/200), show the spectra to be dominated by single peak corresponding to dipolar interaction between the particles. The absorption bands are broad and become asymmetric with increasing volume fraction of the metal. Increase in copper volume fraction showed a red-shift of the surface plasmon peak position due to increase in the bulk-like behaviour due to 


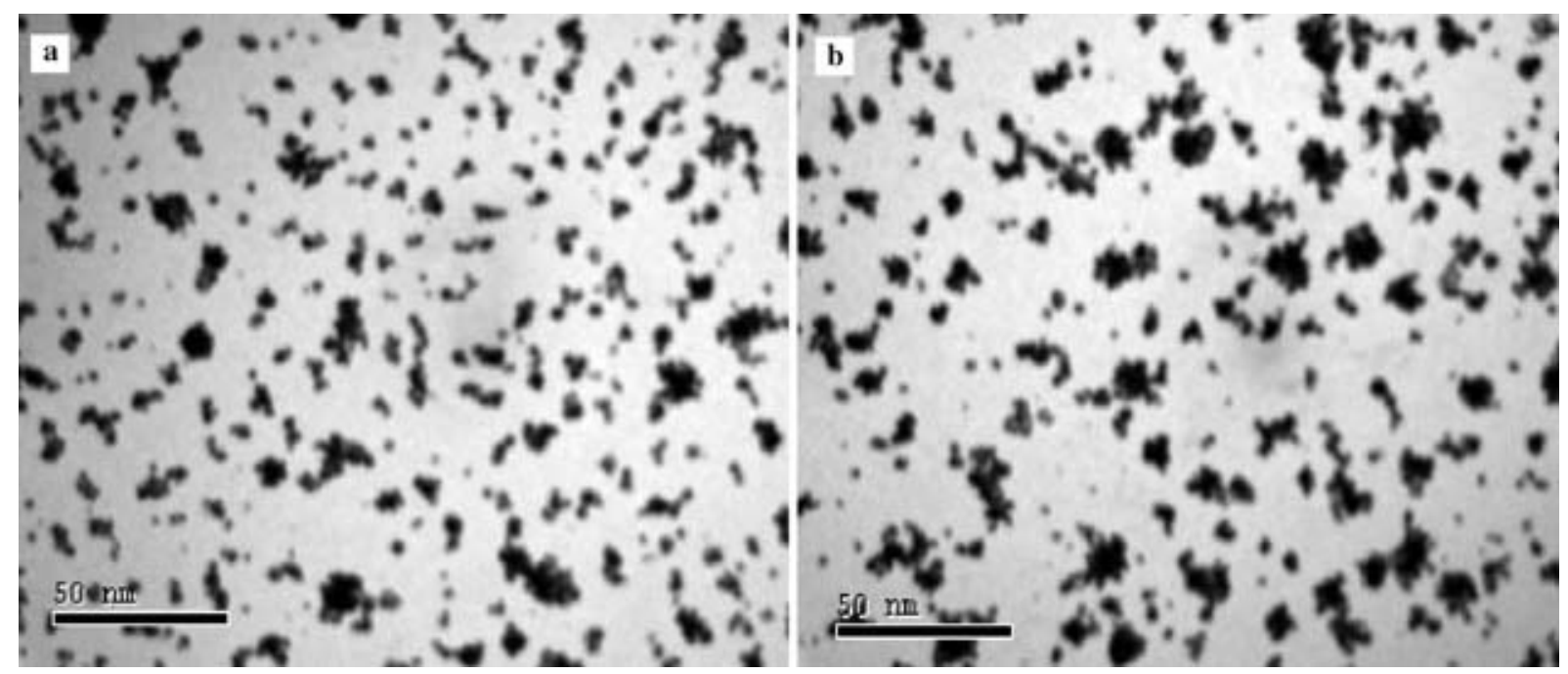

Figure 2. TEM micrographs of composite films at a. $1.5 \mathrm{~V}$ and b. $3.5 \mathrm{~V}$ (Scale: black line represents $50 \mathrm{~nm}$ ).

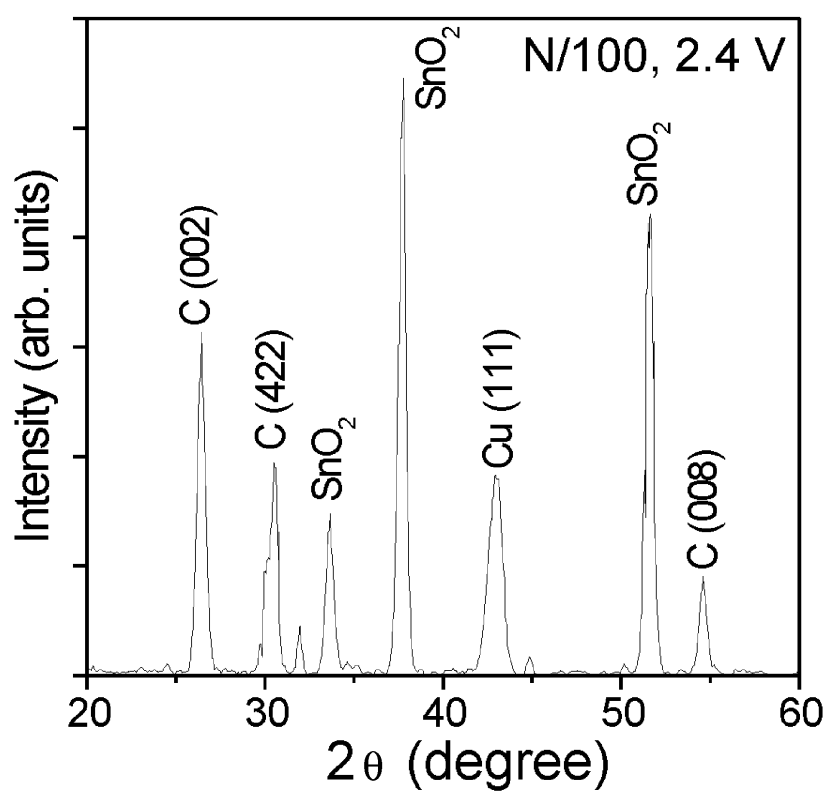

Figure 3. XRD pattern of a representative film.

agglomeration and is a characteristic feature of composite films having large volume fractions of metal nanoparticles. Blue-shift (figure 4a) of the plasmon band with reduction in nanocrystallite size was observed. No plasmon peak appeared for films deposited at voltage $>2.4 \mathrm{~V}$ (figure $4 b$, curves 4 and 5).

\subsection{Explanation using Mie theory}

As per Mie Theory, the optical extinction, $\sigma_{\text {ext }}$, is given by

$$
\sigma_{\mathrm{ext}}(\lambda)=18 \pi f \varepsilon_{0}^{3 / 2} \frac{\varepsilon_{2}(\omega)}{\lambda\left[\left(2 \varepsilon_{0}+\varepsilon_{1}(\omega)\right)^{2}+\varepsilon_{2}^{2}\right]}
$$

with

$$
\begin{aligned}
& \varepsilon_{1}(\omega)=\varepsilon_{1}^{\prime}(\omega)+1-\frac{\omega_{\mathrm{p}}^{2}}{\omega^{2}+\omega_{\mathrm{c}}^{2}}, \\
& \varepsilon_{2}(\omega)=\varepsilon_{2}^{\prime}(\omega)+\frac{\omega_{\mathrm{p}}^{2} \omega_{\mathrm{c}}}{\omega\left(\omega^{2}+\omega_{\mathrm{c}}^{2}\right)}, \\
& \omega_{\mathrm{c}}=\frac{\nu_{\mathrm{f}}}{L}+\frac{2 \nu_{\mathrm{f}}}{d} \text { and } \omega=\frac{2 \pi c}{\lambda},
\end{aligned}
$$

where $\varepsilon_{1}^{\prime}(\omega)$ and $\varepsilon_{2}^{\prime}(\omega)$ are the real and imaginary parts of the dielectric constant of the bulk copper, respectively, $\omega_{\mathrm{p}}$ the plasma frequency, $L$ the electron mean free path in bulk metal, $c$ the velocity of light in vacuum $\left(3 \times 10^{8} \mathrm{~m} / \mathrm{s}\right)$ and $v_{\mathrm{f}}$ the velocity of conduction electron at the Fermi level. Figure 5 shows the experimental and Mie simulated absorbance spectra for the films deposited at (a) a fixed voltage but at different bath concentrations and (b) fixed bath concentration but at different applied voltages. The plasmon-derived resonance peak of copper nanocrystallites become stronger and broader with increase in the metal volume fraction. The resonance peak shifts towards higher wavelength with the increase in loading of copper nanocrystals in DLC matrix. The Mie (1908) theory describes the experimental observation quite faithfully with a slight departure in the higher wavelength region. For copper nanoparticles, we have used the values of $v_{\mathrm{f}}=1.57 \times$ $106 \mathrm{~ms}^{-1}, \mathrm{~L}=39 \mathrm{~nm}$ and $\omega_{\mathrm{p}}=1.32 \times 1016 \mathrm{~s}^{-1}$ with $d, \varepsilon_{\mathrm{m}}$ and $f$ as fitting parameters. The fitted value of the particle size agreed well with that of the peak value of the particle size distribution. 

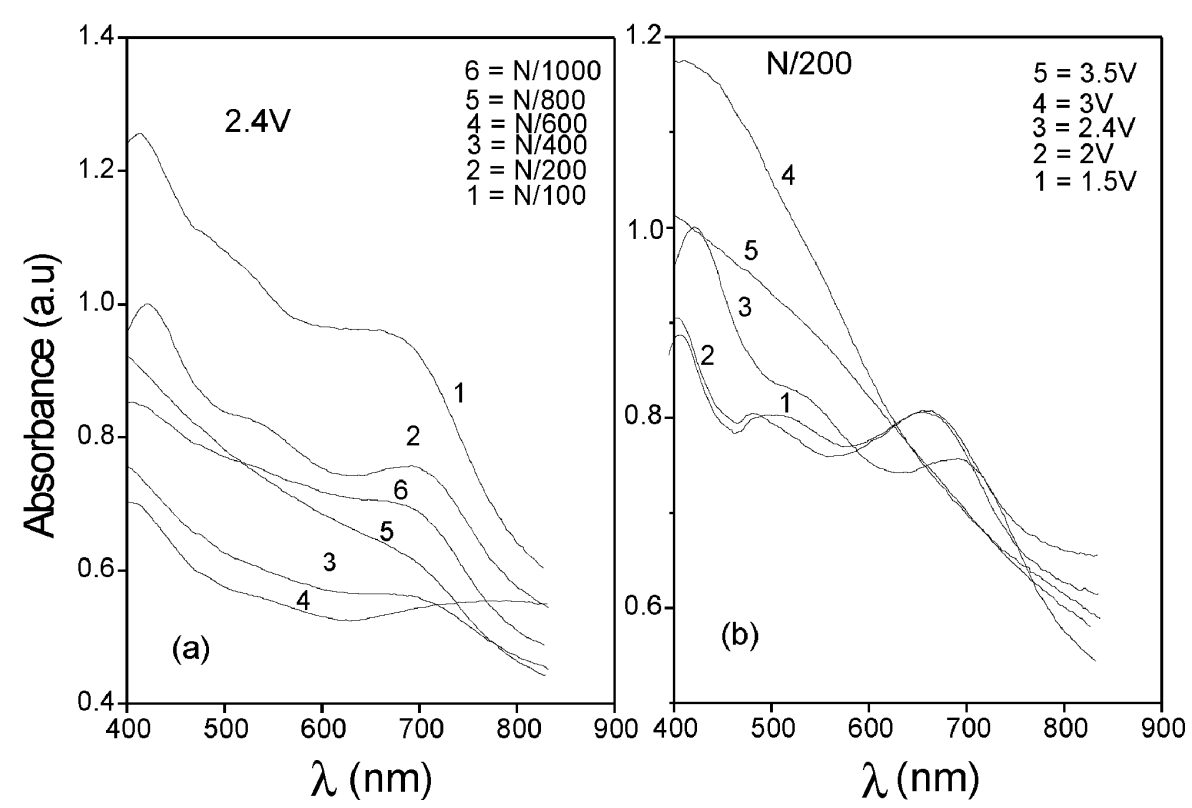

Figure 4. Optical absorption spectra: (a) for concentration variation and (b) for voltage variation.

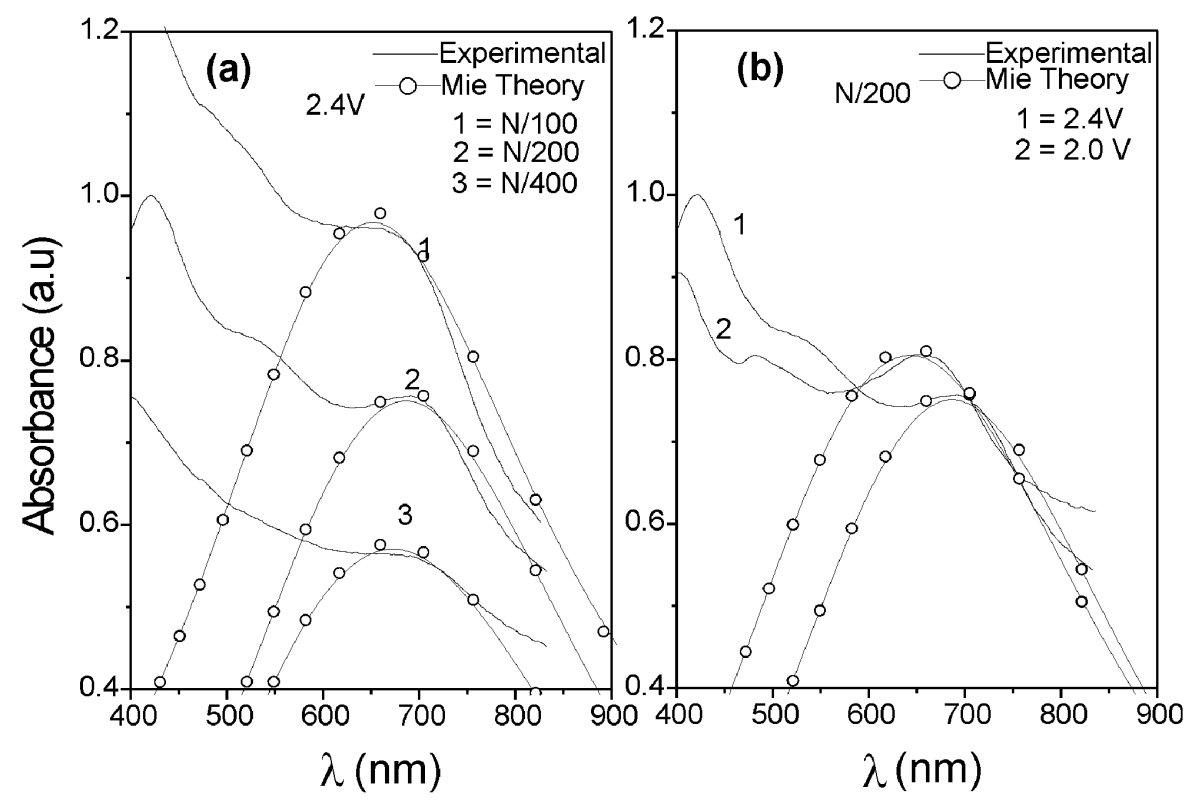

Figure 5. Simulated optical absorption spectra using Mie theory: (a) for concentration variation, fitting parameters are: (1) $d=2.5 \mathrm{~nm}, f=0 \cdot 3, \varepsilon_{\mathrm{m}}=12 \cdot 5$; (2) $d=2 \mathrm{~nm}, f=0 \cdot 1$, $\varepsilon_{\mathrm{m}}=14$; (3) $d=1.5 \mathrm{~nm}, f=0.07, \varepsilon_{\mathrm{m}}=14$ and (b) for voltage variation, fitting parameters are: (1) $d=2 \mathrm{~nm}, f=0 \cdot 1, \varepsilon_{\mathrm{m}}=14$; (2) $d=1.7 \mathrm{~nm}, f=0 \cdot 8, \varepsilon_{\mathrm{m}}=14$.

\subsection{Explanation using $M-G$ theory}

The optical absorbance behaviour of the composite films can be demonstrated qualitatively by effective medium theory predicted by Maxwell-Garnett (M-G theory) valid strictly in the limit of $d / \lambda<0 \cdot 1$ with very small interparticle distances generalized to various shapes of the particles (Maxwell-Garnet 1904). The effective complex dielectric constant, $\varepsilon_{\mathrm{c}}$, for a composite system containing metal particles embedded in a host matrix is defined by

$$
\varepsilon_{\mathrm{c}}=\varepsilon_{0} \frac{\varepsilon_{\mathrm{m}}(1+2 f)+2 \varepsilon_{0}(1-f)}{\varepsilon_{\mathrm{m}}(1-f)+\varepsilon_{0}(2+f)}
$$




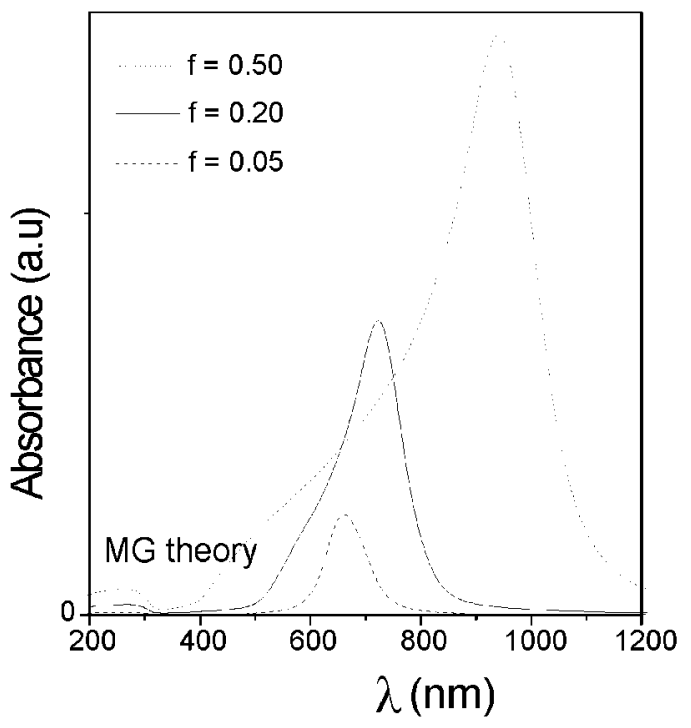

Figure 6. Simulated optical absorption spectra using $\mathrm{M}-\mathrm{G}$ theory.

where $\varepsilon_{\mathrm{c}}^{\prime}(\omega)$ and $\varepsilon^{\prime \prime}{ }_{\mathrm{c}}(\omega)$ are the real and imaginary parts of the complex dielectric constant of the composite system, respectively, $\varepsilon_{\mathrm{m}}$ and $\varepsilon_{0}$ are the dielectric constants of the metal and host matrix, respectively and $f$ the packing fraction of the metal particles. The complex dielectric constant, $\varepsilon_{\mathrm{c}}$, the refractive index, $n_{\mathrm{c}}$ and absorption coefficient, $k_{\mathrm{c}}$, are related as

$$
\begin{aligned}
& \varepsilon_{\mathrm{c}}=n_{\mathrm{c}}^{2}=\varepsilon_{\mathrm{c}}^{\prime}+i \varepsilon_{\mathrm{c}}^{\prime \prime}, \\
& \varepsilon_{\mathrm{c}}^{\prime}=n_{\mathrm{c}}^{2}-k_{\mathrm{c}}^{2}, \varepsilon_{\mathrm{c}}^{\prime \prime}=2 n_{\mathrm{c}} k_{\mathrm{c}} .
\end{aligned}
$$

The absorption maximum occurs at wavelength satisfying

$$
(1-f) \varepsilon_{\mathrm{m}}+(2+f) \varepsilon_{0}=0,
$$

The theoretically generated spectra (figure 6) using the above equation show a shift in the absorption band maxima with an increase of metal phase with change in the intensity and do not fit with our experimental absorbance spectra (figure 4). The modified theory, the dynamical M-G (DMG) (Hornyak et al 1997) theory may give a better explanation and our results on it shall be reported elsewhere.

\section{Conclusions}

Nanocrystalline composite copper/DLC films of various volume fractions with a very narrow size distribution were studied. The optical absorption surface plasmon peak shows a red shift with increase in particle volume fraction. Decrease in particle size shows a blue-shift. Surface plasmon resonance spectra have been successfully explained by Mie theory.

\section{References}

Angus J C, Wang Y and Sunkara M 1991 Ann. Rev. Mater. Sci. 21221

Grigson C W B and Barton E 1967 Br. J. Appl. Phys. 18175

Gupta S et al 2003 Mater. Lett. 573479

Hornyak G L, Patrissi C J and Martin C R 1997 J. Phys. Chem. B101 1548

Maxwell-Garnett J C 1904 Philos. Trans. R. Soc. London 203 385

Mie G 1908 Ann. Phys. Lpz. 25377

Pauleau Y and Thiery F 2002 Mater. Lett. 561053 